\title{
Nalidixic Acid Susceptibility Test for Screening Salmonella Isolates of Reduced Susceptibility/Higher Minimum Inhibitory Concentration to Ciprofloxacin
}

\author{
P. Agrawal' $\mathbf{1}$ R. Tuladhar ${ }^{\mathbf{1}}$ and N. Dahal ${ }^{2}$ \\ ${ }^{1}$ Central Department of Microbiology, Tribhuvan University, Kathmandu, Nepal \\ ${ }^{2}$ Pathology Department, B\&B Hospital, Lalitpur, Nepal \\ e-mail:poohagrawal56@gmail.com
}

\begin{abstract}
Enteric fever is the major diagnosis among febrile patients in Nepal with yearly increase in nalidixic acid resistance and reduced ciprofloxacin susceptibility among Salmonella isolates. This study was carried out to evaluate the validity of nalidixic acid resistance as an indicator of reduced susceptibility of Salmonella isolates to ciprofloxacin. In this study, 999 blood specimens collected from suspected enteric fever patients visiting B\&B Hospital were processed by standard microbiological techniques. Isolates were identified by biochemical tests and serotyping. Antibiotic susceptibility test was performed by Kirby Bauer disc diffusion method and CLSI recommended interpretive criteria. MIC of ciprofloxacin was determined by agar dilution method. Isolation rate of Salmonella species was $6.21 \%$. Among 62 Salmonella isolates, 51 were $S$. typhi, 10 were $S$. paratyphi A and one isolate was $S$. paratyphi B. Only one isolate of $S$. typhi was multi-drug resistant. Resistance to ceftriaxone, cefixime and azithromycin was nil. On disc diffusion test, 55 isolates were resistant to nalidixic acid. Fifty-seven isolates were found to have increased $(\geq 0.125 \mathrm{mg} / \mathrm{ml})$ MIC of ciprofloxacin with the clinical and laboratory standards institute breakpoints. Nalidixic acid resistance showed a predictive value of $100 \%$ for ciprofloxacin resistance. Screening with nalidixic acid disc had a sensitivity of $100 \%$ and a specificity of $71.43 \%$ for the determination of decreased ciprofloxacin susceptibility. Before using ciprofloxacin for the treatment of enteric fever, appropriate identification of Salmonella isolates with reduced ciprofloxacin susceptibility is essential to limit the possible treatment failure and further development of highly resistant strains.
\end{abstract}

Key words: decreased ciprofloxacin susceptibility, enteric fever, MIC value, nalidixic acid resistance

\section{Introduction}

High prevalence of multi-drug resistant Salmonella typhi and $S$. paratyphi A strains during 1990s brought fluoroquinolones into picture for the management of enteric fever (Bhan et al. 2005, Threlfall et al. 1992). Subsequently, increased rates of Salmonella enterica strains with reduced susceptibility to fluoroquinolones (Molbak et al. 2002, Renuka et al. 2004) progressed into high-level resistance to these drugs (Acharya et al. 2009, Saha et al. 2006) have been reported.

Salmonella isolates with decreased ciprofloxacin susceptibility appeared susceptible by routine disc diffusion tests (Asna et al. 2003). Identification of such strains requires susceptibility tests, which give actual minimum inhibitory concentrations. Routine application of these tests for each strain is not convenient, and the literature suggests that resistance to nalidixic acid may be an indicator of decreased susceptibility to ciprofloxacin (Asna et al. 2003, Ercis et al. 2006, Hakanen et al. 1999, Kapil et al. 2002).

Appropriate identification of such population is a matter of concern since such isolates may result in treatment failure (Crump et al. 2003, Shirakawa et al. 2003, Threlfall et al. 2001) and become highly resistant 
upon acquisition of further mutations (Cebrian et al. 2003, Gaind et al. 2006). This research is an attempt to evaluate nalidixic acid screening test as a tool to determine decreased susceptibility (increased MIC) of Salmonella isolates to ciprofloxacin.

\section{Methodology}

The study was conducted prospectively at B\&B Hospital on clinically defined enteric fever patients requesting for blood culture and antibiotic susceptibility testing from April to September 2013. A total of 999 blood samples were studied which included patients of one month old to 97 years old of both sexes. Blood samples were processed by selective enrichment in Tryptone Soya Broth (TSB). Incubation and subcultures on blood agar and MacConkey agar were done as per the standard methods (Collee \& Marr 1996). Suspected colonies were further processed and identified by gram's staining followed by required biochemical tests and confirmed by group and type specific Salmonella antisera (Denka Seiken Co. Ltd., Tokyo, Japan). All the Salmonella spp. isolated from blood samples were subjected for antibiotic susceptibility test by Kirby Bauer disc diffusion method in compliance with CLSI guidelines on Mueller Hinton agar plates using ampicillin $(10 \mu \mathrm{g})$, azithromycin $(15 \mu \mathrm{g})$, cefixime $(5 \mu \mathrm{g})$, ceftriaxone $(30$ $\mu \mathrm{g})$, chloramphenicol $(30 \mu \mathrm{g})$, ciprofloxacin $(5 \mu \mathrm{g})$, co-trimoxazole $(25 \mu \mathrm{g})$ and nalidixic acid $(30 \mu \mathrm{g})$ (HiMedia Laboratories Pvt. Ltd., Mumbai, India). Escherichia coli ATCC 25922 was used for quality control. MIC of ciprofloxacin was determined by agar dilution method following CLSI guidelines (CLSI, 2013). Escherichia coli ATCC 25922 and Staphylococcus aureus ATCC 25923 were used as quality control strains. All the data collected were analyzed using statistical software SPSS version 16.0. The chi-square $\left(\chi^{2}\right)$ test was used to determine the significance of differences. Nalidixic acid susceptibility data (with observed inhibition zone diameter) and MIC values of ciprofloxacin were analyzed by WHONET 5.6 software. Nalidixic acid validation test was carried out by using scatter-plot analysis against ciprofloxacin, and sensitivity and specificity determination. The sensitivity and specificity in determining nalidixic acid resistance by disc testing, with respect to MIC values of ciprofloxacin, were calculated using formulae: $\%$ Sensitivity $=$ true resistant $/$ (true resistant + false sensitive $) \times 100$, and $\%$ Specificity $=$ true sensitive / (false resistant + true sensitive $) \times 100$. The procedures followed were in accordance with the ethical standards of the Ethical Review Board at Institutional Review Committee (IRC) on human experimentation at B\&B Hospital.

\section{Results and Discussion}

Among 999 patients studied during 6 months period, $62(6.21 \%)$ were confirmed as enteric fever cases. Of 62 isolates, $51(82.26 \%)$ were identified as $S$. Typhi, $10(16.13 \%)$ as $S$. Paratyphi A, and one isolate $(1.61 \%)$ as $S$. Paratyphi B. S. Paratyphi C was not isolated. The distribution of these serotypes also varied among different age groups (Table 1).

Table 1. Distribution of the suspected febrile cases and enteric fever cases in different age group

\begin{tabular}{l|l|c|lll}
\hline S.H. & Age group & Total suspected & \multicolumn{3}{|c}{ Total enteric fever pathogens } \\
\cline { 3 - 6 } & (years) & febrile cases & \multicolumn{1}{c}{ Isolated(\%) } & S. Typhi isol ates & S. Paratyphi isol ate \\
\hline 1. & 5 & 101 & $1(0.99)$ & 1 & - \\
2. & $5-18$ & 200 & $28(14)$ & 26 & 2 \\
3. & $19-45$ & 420 & $30(7.14)$ & 22 & 8 \\
4. & $>45$ & 278 & $3(1.08)$ & 2 & 1 \\
& Total & 999 & $62(6.21)$ & 51 & 11 \\
\hline
\end{tabular}

Table 1 displays the distribution of the suspected febrile cases and enteric fever cases in different age groups. Although the overall growth rate was only $6.21 \%$, the percentage of growth in the age group 5-18 years was $14 \%$ followed by $7.14 \%$ in the age group $19-45$ years. The difference in enteric fever cases by age group was statistically significant $(\mathrm{P}<0.05)$.

Male preponderances were seen in infections caused by both the organisms (male: female ratio $=32: 19$ for $\mathrm{S}$. typhi and 6:5 for S. Paratyphi, i.e., 19:12 for all Salmonella isolates) (Table 1). The gender-wise difference was statistically insignificant $(\mathrm{P}>0.05)$.

The most number of suspected febrile cases were observed in the months of July and August and culture positive cases were also greater at those months as $6.25 \%$ and $7.00 \%$ respectively (Fig. 1). 


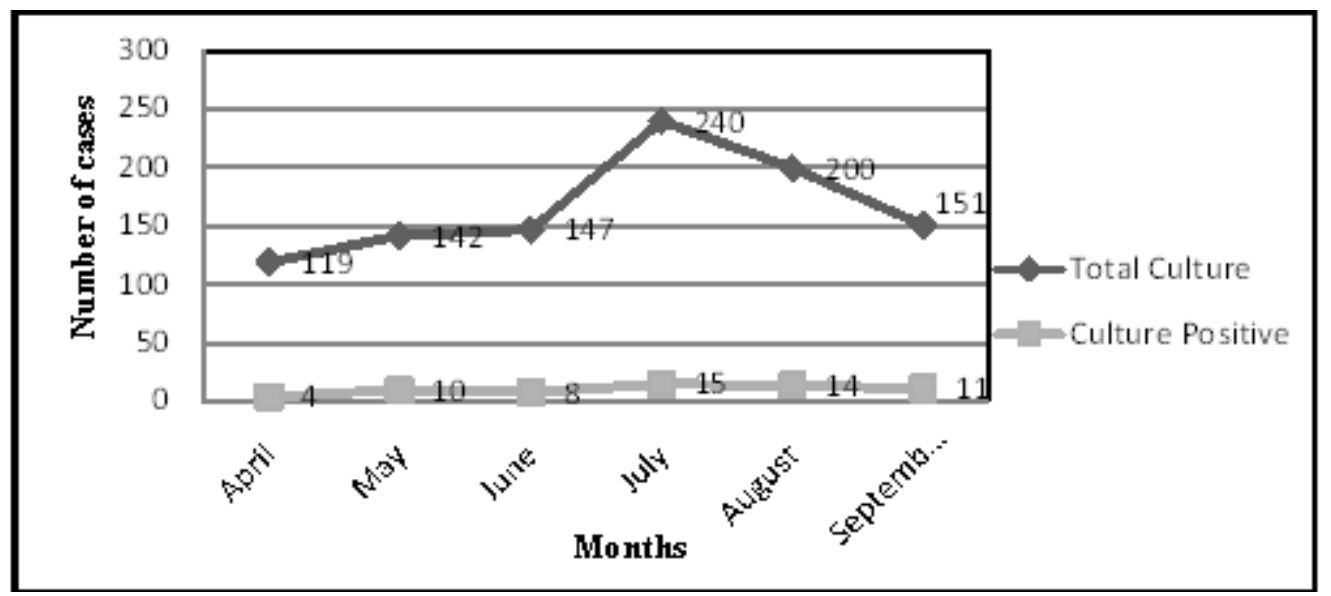

Fig. 1. Pattern of blood culture positive cases at different months

Fig. 2 shows antimicrobial resistance pattern of Salmonella isolates. S. Typhi showed 100\% susceptibility to azithromycin, cefixime and ceftriaxone followed by $98 \%$ susceptibility to chloramphenicol and cotrimoxazole. Resistance to ampicillin and ciprofloxacin was found to be $31.4 \%$ and $80.4 \%$ respectively. Moreover, one isolate $(2.0 \%)$ among S. Typhi was found to be multidrug resistant (MDR) being simultaneously resistant to first line drugs like ampicillin, chloramphenicol and cotrimoxazole (ACCo).

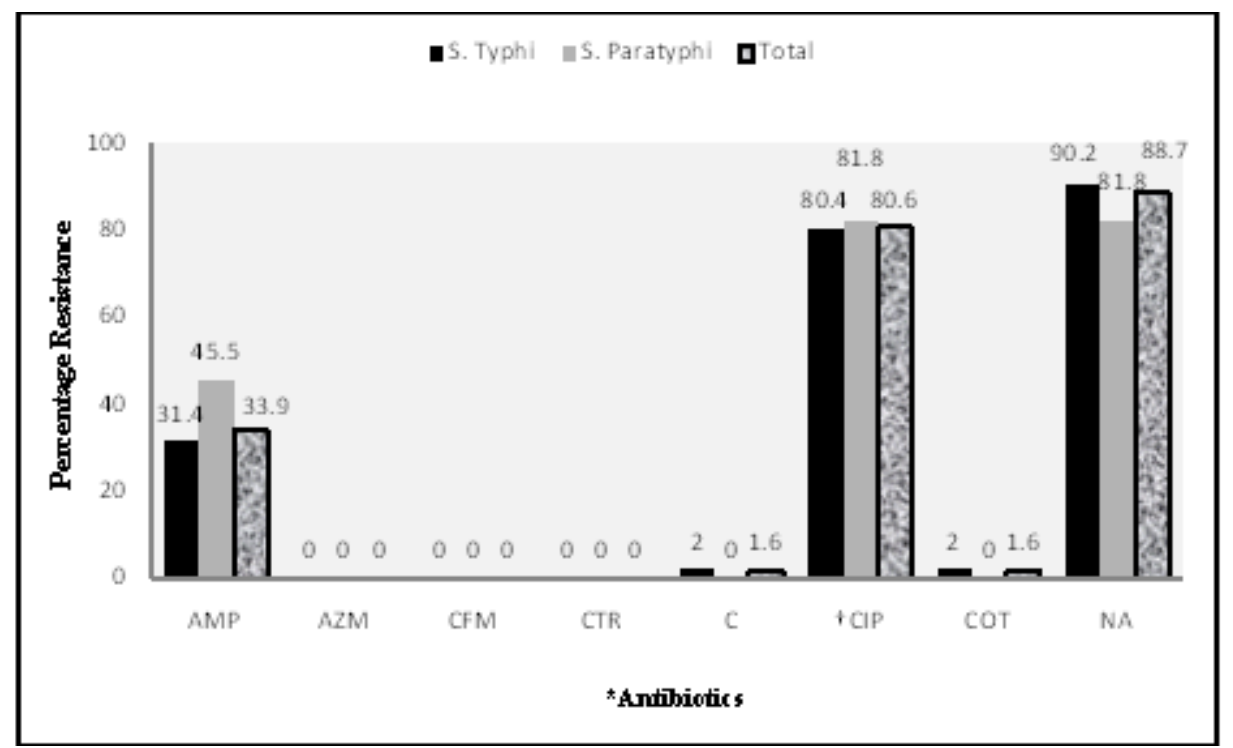

Fig. 2. Antibiotic resistance pattern in S. typhi and S. paratyphi

* AMP (Ampicillin); AZM (Azithromycin); CFM (Cefixime); CTR (Ceftriaxone); C (Chloramphenicol); CIP (Ciprofloxacin); COT (Cotrimoxazole); NA (Nalidixic acid).

$\dagger$ Intermediate resistance observed with ciprofloxacin has been illustrated as resistance in the figure.

Similarly, S. Paratyphi demonstrated $100 \%$ susceptibile to azithromycin, cefixime, ceftriaxone, chloramphenicol and cotrimoxazole. Resistance of isolates towards ampicillin and ciprofloxacin was found to be $45.5 \%$ and $81.8 \%$ respectively. By contrast with S. Typhi, none of S. paratyphi were MDR. 
The overall nalidixic acid resistance rate was quite high $(88.7 \%)$. S. Typhi strains showed even higher $(90.2 \%)$ resistance towards nalidixic acid than $\mathrm{S}$. Paratyphi $(81.8 \%)$ The difference in nalidixic acid resistance among $\mathrm{S}$. Typhi and S. Paratyphi A, however, was statistically insignificant $(\mathrm{P}>0.05)$.

MIC value of ciprofloxacin was determined for 62 isolates, 55 NAR and 7 NAS Salmonella isolates (Figure 3). The $5(8.07 \%)$ isolates had MIC $<0.125$ $\mathrm{mg} / \mathrm{ml}$, and $57(91.93 \%)$ were found to have increased
MIC $(\geq 0.125 \mathrm{mg} / \mathrm{ml})$ of ciprofloxacin. Among 55 NAR isolates (zone of inhibition $\leq 13 \mathrm{~mm}$ ) by disc testing, 32 showed full resistance to ciprofloxacin, 23 exhibited intermediate resistance and none were fully susceptible. Of the 7 NAS isolates (zone of inhibition $\geq 19 \mathrm{~mm}$ ), 5 were fully susceptible to ciprofloxacin and two exhibited reduced ciprofloxacin susceptibility. Thus, screening with $30 \mu \mathrm{g}$ nalidixic acid discs had a sensitivity of $100 \%$ and a specificity of $71.43 \%$ for determination of Salmonella isolates with decreased susceptibility (increased MIC) to ciprofloxacin.

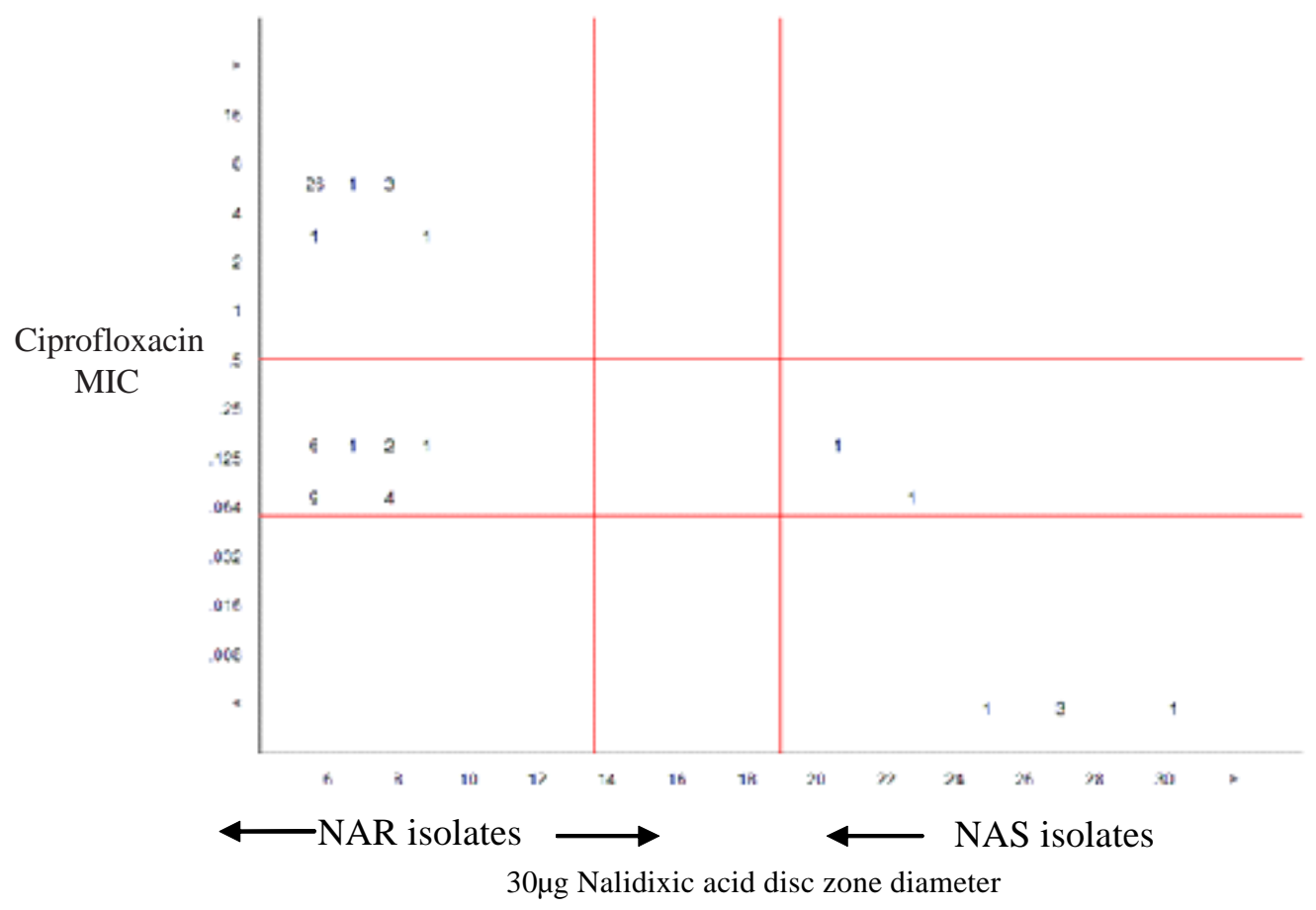

Fig.3. Scatter-plot for MIC values of ciprofloxacin against inhibition zone diameter of nalidixic acid (output from WHONET 5.6 after analysis): Current susceptibility interpretive criteria for ciprofloxacin MIC (d"0.064 for susceptible and e"1 for resistant) and nalidixic acid inhibition zone diameter (d"13 $\mathrm{mm}$ for resistance and e" $19 \mathrm{~mm}$ for susceptible) are shown by parallel lines in the figure.

Growth positive rate of Salmonella species in this study was $6.21 \%$. Similar positive rates have also been reported by Acharya (2008), and Shrestha (2011) which was $9.1 \%$ and $4.69 \%$ respectively. In contrast, high positive rate $(19.28 \%)$ has been reported by Kunwor (2007) from Kathmandu. Relatively low culture positive rate observed in this study might be due to the use of antibiotics prior to blood collection for culture. S. typhi (82.26\%) was more commonly isolated than S. paratyphi $(17.74 \%)$ in the present study. Based on the previous reports, an estimated one case of paratyphoid fever occurs for every four cases of typhoid fever (Crump et al. 2004). This estimate was seen in this study.

Despite the majority, $420(42.0 \%)$ of the suspected cases fell in age group 19-45 years, higher percentage of enteric fever cases (14\%) were observed among 518 years consisting of only 200 samples. In a recent study carried out in five Asian countries, $75 \%$ of the 
growths were from the age group of 5-15 years (Ochiai et al. 2008). The particular age group seems to be more vulnerable to exposure as they may not be having enough care for their foods and potable drinking water due to their busier schedule than that of other age groups. Salmonella infection has been found predominantly in males $(61.3 \%)$ in this study. Previous reports (Acharya 2008, Khanal et al. 2007) from Nepal have also shown higher prevalence of Salmonella infection in males than in females. More outdoor exposure of males has been given the possible reason for higher positive rate among them.

The maximum occurrence of Salmonella infection was observed in July and August, the months that had the temperature and rainfall relatively high. This may be possibly due to the sewage-mediated contamination of water samples during the rainy seasons. Faecal contamination of urban water supplies in Nepal has been reported. An outbreak of $S$. Typhi infecting 5,936 people in Bharatpur in 2002 was traced to the municipal water supply (Lewis et al. 2005).

In this study, resistance to traditional first-line antibiotics such as chloramphenicol and cotrimoxazole has significantly decreased to $1.6 \%$ in comparison to previous report by Khanal et al. (2007) who reported $27.2 \%$ resistance to each antibiotic. But fluoroquinolones which are so frequently used nowadays in empirical treatment of enteric fever showed higher resistance pattern to ciprofloxacin. All strains with reduced susceptibility to ciprofloxacin were fully susceptible to cephalosporins such as ceftriaxone and cefixime. There have been sporadic reports of high-level resistance to ceftriaxone among S. Typhi and S. Paratyphi A (Saha et al. 2006). Although these strains are very rare. Pokharel et al. (2006) has reported ceftriaxone resistance and ESBL production by S. Paratyphi A in Nepal.

In this study, overall nalidixic acid resistance rate was quite high $(88.7 \%)$. S. Typhi strains showed even higher $(90.2 \%)$ resistance towards nalidixic acid than $S$. Paratyphi (81.8\%) which is in agreement with the finding of Acharya (2008). In addition to the nalidixic acid resistance, this study found resistance of Salmonella strains to ampicillin (31.4\% S. Typhi and $45.5 \%$ S. Paratyphi A). In a study done in Chitwan, Acharya et al. (2012) reported very high percentage of ampicillin-resistant isolates (70.6\% S. Typhi and
$78.3 \%$ S. Paratyphi A). It indicates that ampicillin will have very limited value in treating enteric fever. Only one strain of S. Typhi met the conventional definition of multi-drug resistant (MDR) being simultaneously resistant to ampicillin, chloramphenicol and cotrimoxazole (ACCo). An interesting observation in the present study is that MDR in S. Typhi has come down to $1.96 \%$ in comparison to previous reports given by Acharya et al. (2012); Khanal et al. (2007); and Pokharel et al. (2006) which were found to be $16.66 \%$, $26.5 \%$ and $5.18 \%$ MDR isolates respectively.

The findings of this study indicate a remarkable decline in the number of MDR isolates. This was accompanied by an increase in non-MDR isolates, though the majority of these $(88.7 \%)$ was NAR and showed reduced susceptibility to ciprofloxacin. These findings are most likely due to decreased prescribing of traditional antimicrobial agents and an increasing reliance on ciprofloxacin as the first-line treatment for S. Typhi and S. Paratyphi. Reports from most of the countries (Maskey et al. 2008, Saha et al. 2002, Weill et al. 2007) showed the incidence of MDR isolates appears to have decreased with significant increase in the isolates with reduced susceptibility to fluoroquinolones.

According to current CLSI recommendation, susceptible, intermediate and resistant breakpoints for ciprofloxacin among Salmonella spp. are $\leq 0.064 \mu \mathrm{g}$ / $\mathrm{ml}, 0.125-0.5 \mu \mathrm{g} / \mathrm{ml}$ and $\geq 1 \mu \mathrm{g} / \mathrm{ml}$ (respective inhibition zone diameter to $5 \mu \mathrm{g}$ ciprofloxacin are $\geq 31 \mathrm{~mm}, 21-30 \mathrm{~mm}$ and $\leq 20 \mathrm{~mm}$ ) (CLSI 2013). Analysis of the MIC values of ciprofloxacin in this study found that $5(8.1 \%)$ isolates were fully susceptible, 25 $(40.3 \%)$ were intermediately resistance and 32 (51.6\%) exhibited full resistant to ciprofloxacin.

The relevance of using the resistance to nalidixic acid as a marker for reduced ciprofloxacin susceptibility was evaluated by comparing the MIC of ciprofloxacin with inhibition zone diameter of nalidixic acid for all the isolates. Among 55 NAR isolates (zone of inhibition $\leq 13 \mathrm{~mm}$ ), MIC values of ciprofloxacin were found to be $0.125 \mu \mathrm{g} / \mathrm{ml}, 0.25 \mu \mathrm{g} / \mathrm{ml}, 4 \mu \mathrm{g} / \mathrm{ml}$ and 8 $\mu \mathrm{g} / \mathrm{ml}$ for $13,10,2$ and 30 isolates respectively. The 23 of the NAR isolates had decreased ciprofloxacin susceptibility and 32 isolates were found to exhibit high-level resistance to ciprofloxacin. Although 7 NAR isolates appeared susceptible to ciprofloxacin by disc 
diffusion method when interpreted according to current CLSI reference (zone of inhibition $\geq 31 \mathrm{~mm}$ ), they showed increased MIC to ciprofloxacin (MIC $\geq 0.125$ $\mu \mathrm{g} / \mathrm{ml}$ ) which demonstrated that all NAR isolates were with reduced susceptibility to ciprofloxacin.

In the present study, the reduced susceptibility to ciprofloxacin in S. Typhi and S. Paratyphi was strongly correlated with resistance to nalidixic acid. The scatterplot correlating the MIC of ciprofloxacin with inhibition zone diameter of nalidixic acid (Figure 3) illustrated the simultaneous presence of nalidixic acid resistance and reduced ciprofloxacin susceptibility. Similar to this finding Lynch et al. (2009) reported from the United States that the NARST isolates, among which $97 \%$ had decreased ciprofloxacin susceptibility.

In this study, out of 7 NAS isolates, $5(71.43 \%)$ were fully susceptible to ciprofloxacin (MIC $<0.125 \mathrm{mg}$ / $\mathrm{ml}$ ) and two exhibited reduced ciprofloxacin susceptibility while all of the NAR isolates were lesssusceptible or resistant to ciprofloxacin (MIC $\geq$ $0.125 \mu \mathrm{g} / \mathrm{ml}$ ). Thus, screening with $30 \mathrm{mg}$ nalidixic acid disc had a sensitivity of $100 \%$ and a specificity of $71.43 \%$ for determination of decreased susceptibility to ciprofloxacin.

In contrast to high sensitivity of nalidixic acid screening test, the emergence of new quinolone resistance pattern in $S$. enterica which are susceptible to nalidixic acid but exhibited reduced susceptibility to ciprofloxacin (Hakanen et al. 2005) was also reported from South Asia. The increase of such strains ( $3.23 \%$ in the present study) may threaten the value of the nalidixic acid disc test to screen for reduced fluoroquinolone susceptibility in salmonellae.

Salmonella strains with decreased susceptibility to fluoroquinolones are of concern due to the increasing number of treatment failures in invasive salmonellosis (Hakanen et al. 1999). In many tropical countries, including Nepal and Indian subcontinent, there is widespread availability and uncontrolled use of fluoroquinolones. As strains that are already exhibiting decreased susceptibility to ciprofloxacin may require fewer exposures to fluoroquinolones to develop highlevel resistance to ciprofloxacin than the strains that are fully ciprofloxacin susceptible (Cebrian et al. 2003, Gaind et al. 2006). The use of fluoroquinolones as first-line drugs for empirical therapy and management of enteric fever in areas where these strains are endemic is questionable and requires an urgent review. Low exposure to fluoroquinolones reducing the selective pressure on a large bacterial population would definitely lessen the likelihood of selecting mutants (Gaind et al. 2006).

\section{Acknowledgements}

The authors are thankful to the patients and technical staff of B\&B Hospital as well as the faculty and staff of Central Department of Microbiology, Kirtipur for their corresponding support for conducting this study.

\section{References}

Acharya, D. 2008. Fluoroquinolone susceptibility pattern of the Salmonella isolates from enteric fever patients visiting to National Public Health Laboratory, Nepal. M.Sc. Dissertation. Central Department of Microbiology, Tribhuvan University. Pp. 45-59.

Acharya, D., S. Malla, D. Bhatta and S.P. Dumre. 2009. Multidrug resistant Salmonella enterica serovar Typhi. J. Nepal Med. Assoc. 48:196-197.

Acharya, A., H.P. Nepal, R. Gautam and S. Shrestha. 2012. Enteric fever pathogens and their antibiotic susceptibility pattern in Chitwan, Nepal. J. Chitwan Med. Col.l 1; 26-30.

Asna, S.M.Z., J.A. Haq and M. Rahman. 2003. Nalidixic acid-resistant Salmonella enterica serovar Typhi with decreased susceptibility to ciprofloxacin caused treatment failure: A report from Bangladesh. Jpn. J. Infect. Dis. 56:32-33.

Bhan, M.K., R. Bahl and S. Bhatnagar. 2005. Typhoid and paratyphoid fever. Lancet 366:749-762.

Cebrian, L., E. Sirvent and J.C. Rodriguez Diaz. 2003. Characterization of Salmonella spp. mutants produced by exposure to various fluoroquinolones. Int. J. Antimicrob Agents. 22:134-139.

CLSI. 2013. Performance standards for antimicrobial susceptibility testing. $23^{\text {rd }}$ Informational Supplement, M100-S23. Clinical and Laboratory Standards Institute, Wayne, PA.

Collee, J.G. and W. Marr. 1996. Culture of bacteria. In: Mackie and McCartney Practical Medical Microbiology (Eds. J.G. Collee, B.P. Marmion, A.G. Fraser and A. Simmons). 14 $4^{\text {th }}$ edn. Churchill Livingstone, New York, Pp.121-124.

Crump, J.A., T.J. Barret, J.T. Nelson and F.J. Angulo. 2003. Re-evaluating fluoroquinolone breakpoints for Salmonella enterica serotype Typhi and for non-Typhi Salmonellae. Clin. Infect. Dis. 37:75-81.

Crump, J.A., S.P. Luby and E.D. Mintz. 2004. The global burden of typhoid fever. Bull. World Health Organ. 82:346-353. 
Ercis, S., B. Erdem, G. Harcelik and D. Gür. 2006. Nalidixic acid resistance in Salmonella strains with decreased susceptibility to ciprofloxacin isolated from humans in Turkey. Jpn. J. Infect. Dis. 59:117-119.

Gaind, R., B. Paglietti, M. Murgia, R. Dawar, S. Uzzau, P. Cappuccinelli, M. Deb, P. Aggarwal and S. Rubino. 2006. Molecular characterization of ciprofloxacinresistant Salmonella enterica serovar Typhi and Paratyphi A causing enteric fever in India. $J$. Antimicrob. Chemother. 58:1139-1144.

Hakanen, A., P. Kotilainen, J. Jalava, A. Siitonen and P. Huovinen. 1999. Detection of decreased fluoroquinolone susceptibility in salmonellae and validation of nalidixic acid screening test. J. Clin. Microbiol. 37:3572-3577.

Hakanen, A.J., M. Lindgren, P. Huovinen, J. Jalava, A. Siitonen and P. Kotilainen. 2005. New quinolone resistance phenomenon in Salmonella enterica: nalidixic acid-susceptible isolates with reduced fluoroquinolone susceptibility. J. Clin. Microbiol. 43:5775-5778.

Kapil, A., K. Renuka and B. Das. 2002. Nalidixic acid susceptibility test to screen ciprofloxacin resistance in Salmonella Typhi. Indian J. Med. Res. 115:49-54.

Khanal, B., S.K. Sharma, S.K. Bhattacharya, N.R. Bhattarai, M. Deb and R. Kanungo. 2007. Antimicrobial susceptibility patterns of Salmonella enterica serotype Typhi in eastern Nepal. J. Health Popul. Nutr. 25:82-87.

Kunwor, R.B. 2007. Nalidixic acid resistant Salmonella with decreased ciprofloxacin susceptibility. M.Sc. Dissertation. Central Department of Microbiology, Tribhuvan University. Pp. 42-49.

Lewis, M.D., O. Serichantalergs, C. Pitarangsi, N. Chuanak, C.J. Mason, L.R. Regmi, P. Pandey, R. Laskar, C.D. Shrestha and S. Malla 2005. Typhoid fever: a massive, single-point source, multidrug-resistant outbreak in Nepal. Clin. Infect. Dis. 40:554-561.

Lynch, M.F., E.M. Blanton, S. Bulens, C. Polyak, J. Vojdani, J. Stevenson, F. Medalla, E. Barzilay, K. Joyce, T. Barrett and E.D. Mintz. 2009. Typhoid fever in the United States, 1999-2006. JAMA 302:859-865.

Maskey, A.P., B. Basnyat, G.E. Thwaites, J.I. Campbell, J.J. Farrar and M.D. Zimmerman. 2008. Emerging trends in enteric fever in Nepal: 9124 cases confirmed by blood culture, 1993-2003. Trans. R. Soc. Trop. Med. Hyg. 102:91-95.

Molbak, K., P. Gerner-Smidt and H.C. Wegener. 2002. Increasing quinolone resistance in Salmonella enterica serotype Enteritidis. Emerg. Infect. Dis. 5:514-515.

Ochiai, R.L., C.J. Acosta, C.M. Danovaro- Hollyday, D. Baiqing, S.K. Bhattacharya, M.D. Agtini, Z.A. Bhutta, D.G. Canh, M. Ali, S. Shin, J. Wain, A.L. Page, M.J. Albert, J. Farrar, R. Abu-Elyzeed, T. Pang, C.M. Galindo, L. von Seidlein, J.D. Clemens and the Domi
Typhoid Study Group. 2008. A study of typhoid fever in five Asian countries: disease burden and implications for controls. Bull. World Health Organ. 86:260-268.

Pokharel, B.M., J. Koirala, R.K. Dahal, S.K. Mishra, P.K. Khadga and N.R. Tuladhar. 2006. Multidrug-resistant and extended-spectrum beta-lactamase (ESBL)producing Salmonella enterica (serotypes Typhi and Paratyphi A) from blood isolates in Nepal: surveillance of resistance and a search for newer alternatives. Int. J. Infect. Dis. 10:434-438.

Renuka, K., A. Kapil, S.K. Kabra, N. Wig, B.K. Das, V.V.S.P Prasad, R. Chaudhry and P. Seth. 2004. Reduced susceptibility to ciprofloxacin and gyrA gene mutation in North Indian strains of Salmonella enterica serotype Typhi and Pharatyphi A. Microb. Drug. Resist. 10:146-153.

Saha, M.R., P. Dutta, S.K. Niyogi, S. Dutta, U. Mitra, T. Ramamurthy, B. Manna and S.K. Bhattacharya. 2002. Decreasing trend in the occurrence of Salmonella enterica serotype Typhi amongst hospitalised children in Kolkata, India during 1990-2000. Indian J. Med. Res. 115:46-48.

Saha, S.K., G.L. Darmstadt, A.H. Baqui, D.W. Crook, M.N. Islam, M. Islam, M. Hossain, S. El Arifeen, M. Santosham and R.E. Black. 2006. Molecular basis of resistance displayed by highly ciprofloxacin-resistant Salmonella enterica serovar Typhi in Bangladesh. $J$. Clin. Microbiol. 44:3811-3813.

Shirakawa, T., B. Acharya, S. Kinoshita, S. Kumagai, A. Gotoh and M. Kawabata. 2006. Decreased susceptibility to fluoroquinolones and gyrA gene mutation in the Salmonella enterica serovar Typhi and Paratyphi A isolated in Kathmandu, Nepal, in 2003. Diagn. Microbiol. Infect. Dis. 54:299-303.

Shrestha, R. 2011. Quinolone resistance in Salmonella isolates from patients visiting Scheer Memorial Hospital, Banepa. M.Sc. Dissertation. Central Department of Microbiology, Tribhuvan University. Pp. 44-55.

Threlfall, E.J., L.R, Ward, B. Rowe, S. Raghupathi, V. Chandrasekaran, J. Vandepitte and P. Lemmens. 1992. Widespread occurrence of multiple drug-resistant Salmonella Typhi in India. Eur. J. Clin. Microbiol. Infect. Dis. 11:990-993.

Threlfall, E.J., J.A. Skinner and L.R. Ward. 2001. Detection of decreased in vitro susceptibility to ciprofloxacin in Salmonella enterica serotypes Typhi and Paratyphi A. J. Antimicrob. Chemother. 48:740-741.

Weill, F.X., H.H. Tran, P. Roumagnac, L. Fabre, N.B. Minh, T.L. Stavnes, J. Lassen, G. Bjune, P.A. Grimont and P.J. Guerin. 2007. Clonal reconquest of antibioticsusceptible Salmonella enterica serotype Typhi in Son La Province, Vietnam. Am. J. Trop. Med. Hyg. 76:1174-1181. 
Nepal Journal of Science and Technology Vol. 15, No.2 (2014) 97-104 